\title{
Design of coupled tuned liquid column gas dampers for multi-mode reduction in vibrating structures
}

This paper is dedicated to the memory of Franz Ziegler

Received: 4 April 2017 / Revised: 20 June 2017 / Published online: 16 December 2017

(C) The Author(s) 2017. This article is an open access publication

\begin{abstract}
Tuned liquid column gas dampers (TLCGD) show excellent energy and vibration absorbing capabilities appropriate for earthquake engineering. The objective of this work is to introduce a new concept of coupled tuned liquid column gas dampers which allow an extended field of applications. In the proposed configuration, several absorbers are connected in a spatial chain generating a multi-degree of freedom damper system which can be tuned to a selected number of structural modes. Because all absorbers vibrate at different natural frequencies simultaneously, the proposed design represents an improvement over conventional TLCGD which can only be tuned to a single structural mode. Another benefit of multi-mode tuning is the significant increase in active liquid mass compared to other damping devices. For a most effective vibration reduction, a numerical tuning process in state space will deliver the free system natural frequency and damping ratio of all absorbers. The capabilities of the new damping device are demonstrated on a simple laboratory structure that is investigated numerically and experimentally. The results illustrate the excellent energy dissipating properties of the proposed setup and emphasize the adequacy of coupled TLGCD for base-isolated structures. A notable benefit of such types of dampers lies in their lack of moving mechanical parts, their cheap and easy implementation into civil engineering structures and low maintenance costs. Possible modifications with respect to natural frequency and even of the damping properties can be performed with little additional expenses, and the additional weight due to the liquid mass may be used as a possible reservoir, e.g., for fire fighting. Apart from that, they exhibit a performance that is comparable to tuned mass dampers.
\end{abstract}

\section{Introduction}

The prevention of excessive or harmful structural vibrations has always been the subject of theoretical or experimental research in many different fields of engineering, and the continuous development has led to a variety of vibration reduction devices, most of which are based on the efficient absorption of vibrational energy. A well-established and commonly applied technique is the application of dynamic vibration absorbers designed to transfer and dissipate energy from critical building modes. This technique is particularly suitable for lightly damped structures prone to resonant vibrations. A widely used model of passive vibration absorber is the tuned mass damper (TMD), which has been applied for decades to many different kinds of structures in all engineering disciplines. There exist, however, alternative absorber systems, like tuned liquid dampers (TLD), also called sloshing motion dampers or tuned liquid column dampers (TLCD). They all comprise the same basic components, a moving mass exposed to restoring forces (spring) and energy dissipating mechanisms (dashpot). In case of base excitation, alternative concepts like base isolation are adequate, because the accumulation of vibration energy is avoided by simply uncoupling the structure base from its surrounding by appropriate elements. This strategy is very effective in earthquake protection because it reduces the energy dissipation

M. J. Hochrainer · P. A. Fotiu ( $\varangle)$

Department of Applied and Numerical Mechanics, University of Applied Sciences, Wiener Neustadt, Austria

E-mail:pf@fhwn.ac.at 
demand of the higher structural vibration modes. However, base isolation of structures results in critical rigid body type modes of vibrations, which require other energy dissipating measures to achieve a high level of protection, such as dynamic absorbers. Although successfully applied to suppress vibrations in high-rise buildings, TMD suffer from the difficulty of ensuring a smooth, frictionless motion of huge masses. While active friction compensation is possible, a common way to overcome friction issues is to vertically suspend the absorber mass on cables, thereby forming a pendulum type system, which is, e.g., installed in the Taipei 101 tower. Since pendulum type absorbers can hardly compensate for torsional vibrations, they are an ideal TMD alternative for symmetric high-rise buildings only.

A different approach to circumvent the TMD friction problems is to substitute the rigid mass by moving fluids. Sloshing motion dampers (TLD) have been successfully applied to reduce wind-induced vibrations of smokestacks or wind turbines. However, difficulties in tuning, variable damping and a comparatively low active mass prevented further successful applications. TLCD are known to overcome these deficiencies by guiding the liquid flow in a rigid piping system. Similar to TMD, the vibration reduction results from a transfer of structural vibration energy into a liquid movement with energy dissipation by viscous and turbulent damping. Several innovative strategies have been developed to improve the tuning of TLCD in practical applications in order to make these devices more competitive [1-3]. Still, TLCD operate at extremely low natural frequencies in the range of $0.1-0.5 \mathrm{~Hz}$, and consequently, the original design is only appropriate for very large structures. However, the development of the modified tuned liquid column gas damper (TLCGD) extended the field of possible applications to structures with critical frequencies up to several $\mathrm{Hz}$, see [4].

Due to their simple design and a performance comparable to TMD, tuned liquid column (gas) dampers have caused increased research efforts, in both analytical and experimental studies [5-7]. The conventional plane V-shaped TLCGD design has been successfully applied to systems with dominating horizontal vibrations. In case of large flexural vibrations, e.g., long span bridges, the novel space optimized pipe-in-pipe design of vertical TLCGD (VTLCGD) renders additional efficient damping [8,9]. Other studies prove their efficiency for providing additional damping for low frequency base isolation modes [10-13,29]. The combination with base isolation is particularly promising, because it permits the installation of the heavy piping system at the base of a structure.

In order to mitigate flexural and torsional vibrations, different configurations have been proposed $[4,9,12$, 14]. A promising approach to improve the performance of TLCGD makes use of active air springs by actively adapting the pressure at the closed pipe sections of the absorber $[15,16]$. After applying a proper control algorithm, the system can be used in both active and passive configurations, and it is possible to increase the effective damping of several modes with a single device.

The salient features of TLCGD include comparatively low installation costs, easy application to new or already existing structures, a simple tuning mechanism, no moving mechanical parts, and therefore negligible maintenance requirements as well as little additional weight if the system is used as a water reservoir, e.g., for the sake of firefighting. The theoretical and experimental research work of the last years has clearly indicated that tuned liquid column dampers are very competitive when compared to the popular TMD of mass spring dashpot type. For a comprehensive overview, see [9]. The profound understanding of TLCD has led to simple design guidelines for optimal absorber geometry, placement and tuning, and so far all studies clearly indicate that TLCGD could replace TMD in many fields of applications. Possible applications include, among others, base-excited asymmetric high-rise buildings, wind, traffic or earthquake-induced bending torsional vibrations of bridges and base-isolated systems. Temporary mobile TLCD units may be used in case of vibration-prone construction processes of bridges and other structures as well $[9,17]$. Real size installations have also been reported, e.g., a TLCD installation in a 26-story, 106-m high hotel in Japan [18], or the 48-story One Wall Center in Vancouver, Canada [19].

Nevertheless, a considerable deficiency when compared to TMD is the incapability of individual TLCGD to mitigate flexural and torsional vibrations simultaneously. Hence, the focus of the present work is directed to an innovative coupled TLCGD which allows the tuning to several modes. While still using the absorber in a passive configuration, this multi-mode tuning is of practical relevance, because it significantly reduces the required fluid mass. This is an important extension over the TLCD, which can be tuned to a single mode only. For example, a series of two coupled dampers may achieve the same effects with only half the active mass of a conventional TLCD.

Finally, it must be said that TLCD like all passive devices reduce vibrations mainly by counteracting the excitations rather than dissipating the induced energy. It will be shown in later chapters that such systems when appropriately tuned work very well in reducing natural vibrations and consequently also resonances. The merits in rather broadbanded excitations remain questionable, since some spectral components might even 


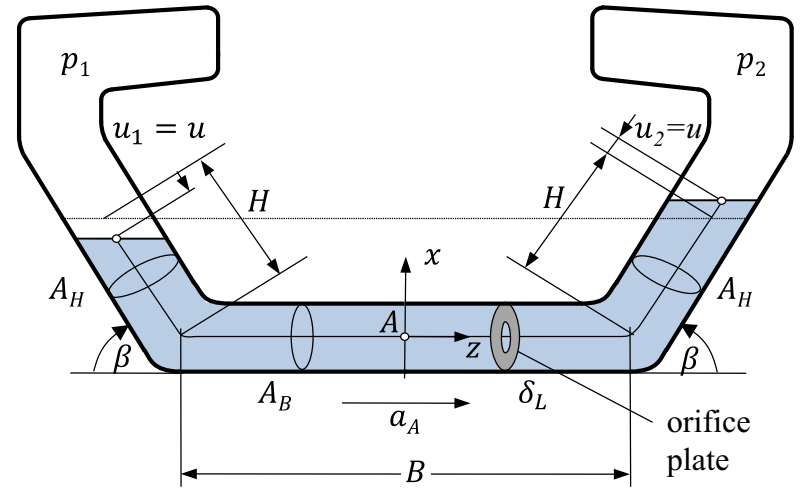

(a)

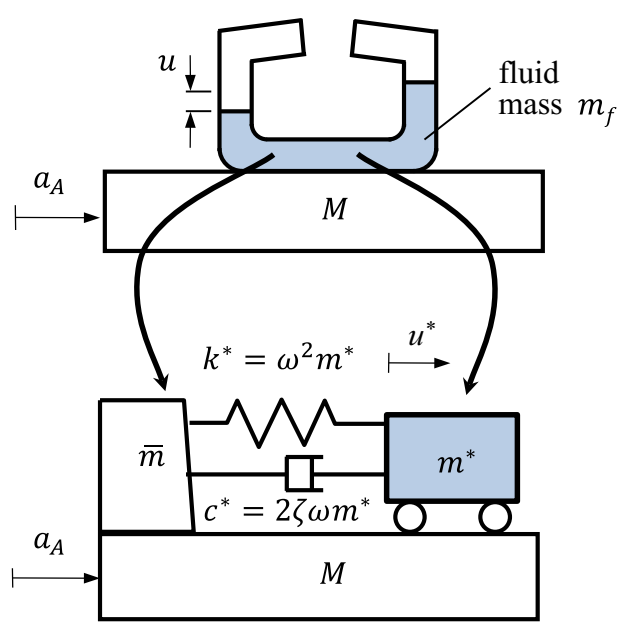

(b)

Fig. 1 a TLCGD of general shape with a relative streamline at a time instant $t$. b TLCGD as an equivalent TMD with active mass $m^{*}$ and dead weight load $\bar{m}=m_{f}-m^{*}$

be enhanced. A study of the performance of such systems under broadband excitation can be found in [20]. Consequently, sustainable designs for earthquake protection should not rely on passive devices such as TLCD or TMD alone, but are complemented by dissipating systems as well.

\section{Description of a TLCGD}

\subsection{Equation of motion}

The movement of the liquid column is governed by the non-stationary Bernoulli equation [21] applied in a moving frame. Although many different TLCGD geometries have been proposed, the main characteristics can be derived from a U- or V-shaped device, see Fig. 1a. The liquid moves inside a horizontal and two symmetric inclined pipe sections of length $B$ and $H$, respectively. We assume piecewise constant cross-sectional areas, $A_{H}, A_{B}$. Frequently, orifice plates are inserted to increase the head loss in the moving fluid.

With $u(t)$ being the amplitude of the liquid surface oscillation and $a_{A}(t)$ the horizontal acceleration of the tube center $A$, the equation of motion reads [22],

$$
\ddot{u}+2 \zeta \omega \dot{u}+\omega^{2} u=-\kappa a_{A},
$$

with $g$ being the constant of gravity, and

$$
\begin{aligned}
\omega & =\sqrt{\frac{g}{L_{0}}}, \\
\zeta & =\frac{2 \lambda}{3 \pi} \frac{\max |u|}{L_{\text {eff }}}, \\
\kappa & =\frac{B+2 H \cos \beta}{L_{\text {eff }}} .
\end{aligned}
$$

The factor $\lambda$ appearing in Eq. (2.2) accounts for the head loss in the fluid, which is given by the nonlinear expression $\Delta p=\lambda \rho|\dot{u}| \dot{u} / 2$. The damping factor $\zeta$ according to Eq. (2.2) results from an equivalent linearization technique, where the nonlinear loss is replaced by an equivalent linear viscous damping term that dissipates the same amount of energy during one cycle. As a consequence, the resulting factor $\zeta$ depends on the vibration amplitude max $|u|$. The geometric parameters appearing in Eq. (2) are defined as follows: 


$$
\begin{aligned}
L_{0} & =\frac{L_{\mathrm{eff}}}{2\left(\sin \beta+h_{0} / H_{a}\right)}, \\
L_{\mathrm{eff}} & =2 H+\frac{A_{H}}{A_{B}} B, \quad h_{0}=\frac{n p_{0}}{\rho g}, \quad H_{a}=\frac{V_{0}}{A_{H}}
\end{aligned}
$$

where $\rho$ is the liquid density, and $V_{0}, p_{0}, n$ denote the gas volume, the equilibrium gas pressure, and the polytropic index, respectively [23]. The factor $h_{0} / H_{a}$ in Eq. (3.1) represents the elastic effect of the gas volume which is acting as a spring. For extremely slow vibrations, the gas spring will operate under isothermal conditions $(n=1)$, while at high frequencies an adiabatic change will occur and the polytropic index becomes $n=1.4$. For all other operating conditions, $n$ is in the range of $1 \leq n \leq 1.4$. The gas spring serves as a convenient means for tuning the TLCGD resulting in an extended range of frequencies when compared to the classical TLCD. Conventional TLCD usually are restricted to frequencies up $0.5 \mathrm{~Hz}$ in practical applications, while the passive gas spring allows the application of TLCGD for frequencies up to about $5 \mathrm{~Hz}$. With respect to the validity of piston theory, the frequency will be limited by the (relative) maximum fluid speed, $\dot{u}=\omega u$, which must remain lower than a critical speed of about $12 \mathrm{~m} / \mathrm{s}$ to avoid cavitation at the fluid-gas interface [8]. Hence, for a given fluid stroke, the practicable frequency range in Eq. (1) is limited.

\subsection{Coupling to a single degree of freedom structure}

If attached to a moving floor, the fluid mass $m_{\mathrm{f}}$ can be separated into an active mass $m^{*}$ which accounts for the inertia effect of the damping device while the remaining fluid mass $\bar{m}$ merely acts as a dead weight, as shown in Fig. 1b. It is shown in $[4,9]$ that

$$
\begin{aligned}
m^{*} & =\kappa \bar{\kappa} m_{\mathrm{f}}, \\
\bar{m} & =m_{\mathrm{f}}-m^{*}, \\
m_{\mathrm{f}} & =\rho A_{H} L_{1},
\end{aligned}
$$

with

$$
\begin{aligned}
\bar{\kappa} & =\kappa \frac{L_{\mathrm{eff}}}{L_{1}}, \\
L_{1} & =2 H+\frac{A_{B}}{A_{H}} B .
\end{aligned}
$$

This allows for an analogy between a TLCGD and a conventional tuned mass damper (TMD), where numerous criteria exist for the optimal choice of the dampers' natural frequency and damping ratio. If a TMD with mass $m$, damping coefficient $\zeta$, and natural frequency $\omega$ is attached to a base-excited single degree of freedom host structure with mass $M$, damping coefficient $Z$ and natural frequency $\Omega$, then the coupled system reads [4]

$$
\left[\begin{array}{cc}
1+\mu & \mu \\
1 & 1
\end{array}\right]\left[\begin{array}{c}
\ddot{w} \\
\ddot{u}
\end{array}\right]+\left[\begin{array}{cc}
2 Z \Omega & 0 \\
0 & 2 \zeta \omega
\end{array}\right]\left[\begin{array}{l}
\dot{w} \\
\dot{u}
\end{array}\right]+\left[\begin{array}{cc}
\Omega^{2} & 0 \\
0 & \omega^{2}
\end{array}\right]\left[\begin{array}{l}
w \\
u
\end{array}\right]=-\ddot{w}_{g}\left[\begin{array}{c}
\alpha+\mu \\
1
\end{array}\right]
$$

where $w(t)$ is the displacement of the structure relative to the base, $\ddot{w}_{\mathrm{g}}$ is the ground acceleration, and

$$
\begin{aligned}
& \mu=m / M, \\
& \alpha=1 .
\end{aligned}
$$

The same equation can be used also for a TLCGD, if the following parameters are replaced by the corresponding starred quantities.

$$
\begin{aligned}
\mu \rightarrow \mu^{*} & =\frac{m^{*}}{M^{*}}, \quad \Omega \rightarrow \Omega^{*}=\frac{\Omega}{\sqrt{M^{*} / M}}, \quad Z \rightarrow Z^{*}=\frac{Z}{\sqrt{M^{*} / M}}, \\
u \rightarrow u^{*} & =u / \kappa,
\end{aligned}
$$

with $m^{*}$ according to Eq. (4.1), and

$$
M^{*}=M+\bar{m} .
$$




\subsection{Coupling to a multi-degree of freedom structure}

In multi-degree of freedom structures, it is frequently sufficient to couple the damper only to the single mode that is to be damped. Then, again, one has to consider merely a two degree of freedom structure, where the structural mass $M$ is replaced by the modal mass $M_{j}$ of the corresponding mode. Consider a plane $N$ degree of freedom structure with mass matrix $\mathbf{M}$, damping matrix $\mathbf{C}$, and stiffness matrix $\mathbf{K}$. If the TLCGD is attached to the $i$-th floor, the equations of motion for the relative horizontal story displacements $\mathbf{w}(t)$ and the damper oscillation $u(t)$ read

$$
\begin{aligned}
\mathbf{M} \ddot{\mathbf{w}}+\mathbf{C} \dot{\mathbf{w}}+\mathbf{K} \mathbf{w} & =-\mathbf{M} \mathbf{r}_{s} \ddot{w}_{\mathrm{g}}+\mathbf{F}_{i}, \\
\ddot{u}+2 \zeta \omega \dot{u}+\omega^{2} u & =-\kappa\left(\ddot{w}_{\mathrm{g}}+\mathbf{s}_{i}^{\mathrm{T}} \ddot{\mathbf{w}}\right)
\end{aligned}
$$

where

$$
\mathbf{F}_{i}=-\mathbf{s}_{i} m_{\mathrm{f}}\left(\ddot{w}_{\mathrm{g}}+\mathbf{s}_{i}^{\mathrm{T}} \ddot{\mathbf{w}}+\bar{\kappa} \ddot{u}\right)
$$

denotes the interaction force between the structure and the damper on the $i$-th floor.

In Eq. (10), $\mathbf{s}_{i}$ is a $(N \times 1)$ unit vector in the $i$-direction, and $\mathbf{r}_{S}$ represents the projection of the ground displacement onto the $N$ degrees of freedom. In case of a frame type structure under a constant horizontal ground movement of all supports, $\mathbf{r}_{S}$ is a $(N \times 1)$ vector with all components equal to 1 . Using the orthogonal modes of the structure $\boldsymbol{\phi}_{n}, n=1, \ldots, N$, Eq. (10.1) can be decomposed into $N$ modal equations. If the TLCGD are designed to reduce the $j$-th mode, we restrict the coupling of Eq. (10.2) to the $j$-th modal equation,

$$
\boldsymbol{\phi}_{j}^{\mathrm{T}} \mathbf{M} \boldsymbol{\phi}_{j} \ddot{q}_{j}+\boldsymbol{\phi}_{j}^{\mathrm{T}} \mathbf{C} \boldsymbol{\phi}_{j} \dot{q}_{j}+\boldsymbol{\phi}_{j}^{\mathrm{T}} \mathbf{K} \boldsymbol{\phi}_{j} q_{j}=-\boldsymbol{\phi}_{j}^{\mathrm{T}} \mathbf{M} \mathbf{r}_{S} \ddot{w}_{\mathrm{g}}-\boldsymbol{\phi}_{j}^{\mathrm{T}} \mathbf{s}_{i} m_{\mathrm{f}}\left(\ddot{w}_{\mathrm{g}}+\mathbf{s}_{i}^{\mathrm{T}} \ddot{\mathbf{w}}+\bar{\kappa} \ddot{u}\right),
$$

where $q_{j}(t)$ is the $j$-th modal coordinate according to the mapping $\mathbf{w}(t)=\sum_{n=1}^{N} q_{n}(t) \boldsymbol{\phi}_{n}$. The last term of Eq. (11) describes the reaction force between the damper and the $j$-th mode. If the structural modes are sufficiently well separated, we may neglect all contributions other than the $j$-th component in $\ddot{\mathbf{w}}$; that is, we set $\ddot{\mathbf{w}} \approx \ddot{q}_{j} \boldsymbol{\phi}_{j}$. Then, we obtain the following coupled system:

$$
\begin{aligned}
& {\left[\begin{array}{cc}
M_{j}+\phi_{j i}^{2} m_{\mathrm{f}} & \bar{\kappa} \phi_{j i} m_{\mathrm{f}} \\
\bar{\kappa} \phi_{j i} m_{\mathrm{f}} & (\bar{\kappa} / \kappa) m_{\mathrm{f}}
\end{array}\right]\left[\begin{array}{c}
\ddot{q}_{j} \\
\ddot{u}
\end{array}\right]+\left[\begin{array}{cc}
2 Z_{j} \Omega_{j} M_{j} & 0 \\
0 & 2 \zeta \omega(\bar{\kappa} / \kappa) \\
m_{\mathrm{f}}
\end{array}\right]\left[\begin{array}{c}
\dot{q}_{j} \\
\dot{u}
\end{array}\right]} \\
& \quad+\left[\begin{array}{cc}
\Omega_{j}^{2} M_{j} & 0 \\
0 & \omega^{2}(\bar{\kappa} / \kappa) m_{\mathrm{f}}
\end{array}\right]\left[\begin{array}{c}
q_{j} \\
u
\end{array}\right] \\
& =-\ddot{w}_{\mathrm{g}}\left[\begin{array}{c}
\boldsymbol{\phi}_{j}^{\mathrm{T}} \mathbf{M} \mathbf{r}_{S}+\phi_{j i} m_{\mathrm{f}} \\
\bar{\kappa} m_{\mathrm{f}}
\end{array}\right]
\end{aligned}
$$

where $\phi_{j i}=\mathbf{s}_{i}^{\mathrm{T}} \boldsymbol{\phi}_{j}$ is the $i$-th component of the mode $\boldsymbol{\phi}_{j}$, and the corresponding modal parameters are

$$
\begin{aligned}
M_{j} & =\boldsymbol{\phi}_{j}^{\mathrm{T}} \mathbf{M} \boldsymbol{\phi}_{j}, \\
\Omega_{j}^{2} & =\left(1 / M_{j}\right) \boldsymbol{\phi}_{j}^{\mathrm{T}} \mathbf{K} \boldsymbol{\phi}_{j}, \\
Z_{j} & =\left(1 / 2 \Omega_{j} M_{j}\right) \boldsymbol{\phi}_{j}^{\mathrm{T}} \mathbf{C} \boldsymbol{\phi}_{j} .
\end{aligned}
$$

Equation (12) can also be transformed into an equivalent TMD system with equations of motion similar to Eq. (6),

$$
\left[\begin{array}{cc}
1+\mu^{*} & \mu^{*} \\
1 & 1
\end{array}\right]\left[\begin{array}{c}
\ddot{q}_{j} \\
\ddot{u}^{*}
\end{array}\right]+\left[\begin{array}{cc}
2 Z_{j}^{*} \Omega_{j}^{*} & 0 \\
0 & 2 \zeta \omega
\end{array}\right]\left[\begin{array}{c}
\dot{q}_{j} \\
\dot{u}^{*}
\end{array}\right]+\left[\begin{array}{cc}
\Omega_{j}^{* 2} & 0 \\
0 & \omega^{2}
\end{array}\right]\left[\begin{array}{c}
q_{j} \\
u^{*}
\end{array}\right]=-\ddot{w}_{\mathrm{g}}^{*}\left[\begin{array}{c}
\alpha^{*}+\mu^{*} \\
1
\end{array}\right]
$$

with

$$
\mu^{*}=\frac{\phi_{j i}^{2} m^{*}}{M^{*}}, \quad M^{*}=M_{j}+\phi_{j i}^{2} \bar{m},
$$


and

$$
\begin{aligned}
\Omega_{j}^{*} & =\frac{\Omega_{j}}{\sqrt{M^{*} / M_{j}}}, \quad Z_{j}^{*}=\frac{Z_{j}}{\sqrt{M^{*} / M_{j}}}, \\
u^{*} & =\frac{u}{\kappa \phi_{j i}}, \quad \ddot{w}_{\mathrm{g}}^{*}=\frac{\ddot{w}_{\mathrm{g}}}{\phi_{j i}}, \\
\alpha^{*} & =\frac{\phi_{j i} \boldsymbol{\phi}_{j}^{\mathrm{T}} \mathbf{M} \mathbf{r}_{S}+\phi_{j i}^{2} \bar{m}}{M^{*}} .
\end{aligned}
$$

\subsection{Tuning of the damping parameters}

For the optimal tuning of a two degree of freedom TMD system, the Den Hartog criterion can be applied [24]. The same formulas may be used for the analogous system (14), which delivers the following estimates of the optimal frequency and damping coefficient of the equivalent TLCGD:

$$
\begin{aligned}
\omega_{\mathrm{opt}} & =\frac{\Omega^{*}}{1+\mu^{*}}=\frac{\Omega}{\left(1+\mu^{*}\right) \sqrt{M^{*} / M}}, \\
\zeta_{\mathrm{opt}} & =\sqrt{\frac{3}{8} \frac{\mu^{*}}{1+\mu^{*}}} .
\end{aligned}
$$

Choosing these parameters, the dynamic magnification factor of the absolute floor acceleration will be minimized under a harmonic ground excitation. According to [25], this setting also minimizes the displacement magnification due to a harmonic force acting at the structural degree of freedom.

\section{Coupling of TLCGD systems}

While a single TLCGD can be optimized to reduce a single mode only, the simultaneous reduction in multiple modes requires a series of TLCGD. These might be either a sum of individual dampers or an arrangement of coupled dampers. By a suitable design of such damping devices, excitations in different directions can be treated simultaneously, e.g., transverse and torsional modes. In the following, we describe a system of two coupled TLCGD's, where the coupling is governed by the gas spring of volume $V_{0}$ between the rigid pipes, as shown in Fig. 2a. Accordingly, the two liquid columns work as a two degree of freedom oscillator with three springs, as shown in Fig. 2b. Note, that location and orientation of the two TLCD can and should be chosen independently within the floor for an optimal performance.

Similarly to the single TLCGD of Eq. (1), we may set up the equations of motion for the coupled device,

$$
\ddot{u}_{k}+\frac{\Delta p_{k}}{\rho L_{\text {eff } k}}+\frac{2 g \sin \left(\beta_{k}\right)}{L_{\text {eff } k}} u_{k}=-\kappa_{k} a_{A k}, \quad k=1,2,
$$

where

$$
\begin{aligned}
L_{\text {eff } k} & =2 H_{k}+\left(A_{H} / A_{B k}\right) B_{k}, \\
\kappa_{k} & =\frac{2 H_{k} \cos \left(\beta_{k}\right)+B_{k}}{L_{\text {eff } k}}, \quad k=1,2,
\end{aligned}
$$

and $a_{A k}$ denotes the acceleration of the respective tube center $A_{k}$. In a series formation, these accelerations can be set equal, while in rotated configurations the values of the $a_{A k}$ are in general different, compare with Fig. 3. Considering the pressure differences, we have according to Fig. 2

$$
\Delta p_{1}=p_{2}-p_{1}, \Delta p_{2}=p_{4}-p_{3} .
$$

Since in a coupled damper $p_{2}=p_{3}$, we have $\Delta p_{1}=-\Delta p_{2}=\Delta p$. Using the equation of state for an ideal gas with volume $V_{0}$ at equilibrium, we obtain for the pressure difference

$$
\Delta p=p_{0}\left[\left(\frac{V_{0}}{V_{0}+\Delta V}\right)^{n}-1\right]
$$




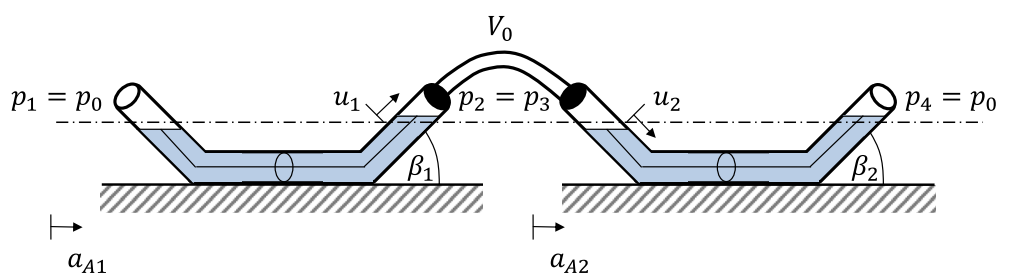

(a)

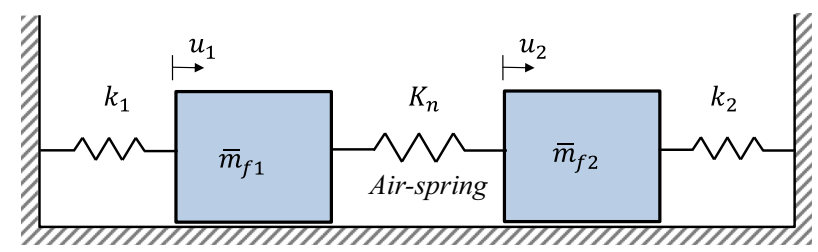

(b)

Fig. 2 a Two coupled TLCGD and $\mathbf{b}$ mechanical model of an equivalent two degree of freedom oscillator. Note that the loading can no longer be applied as a common base excitation

with $\Delta V=A_{H 2} u_{2}-A_{H 1} u_{1}$. Since $\Delta V \ll V_{0}$, we may linearize Eq. (21), and assuming equal areas $A_{H 1}=$ $A_{H 2}=A_{H}$, this results in

$$
\Delta p=\frac{n p_{0}}{V_{0} / A_{H}}\left(u_{1}-u_{2}\right)=\frac{\rho g h_{0}}{H_{a}}\left(u_{1}-u_{2}\right),
$$

with $h_{0}, H_{a}$ according to Eq. (3.2). The coupled TLCGD system acts as a two degree of freedom oscillator with equivalent masses and stiffnesses

$$
\begin{aligned}
\bar{m}_{f k} & =\left(\bar{\kappa}_{k} / \kappa_{k}\right) m_{f k}, k_{k}=2 \rho g A_{H} \sin \beta_{k}, \quad k=1,2, \\
K_{n} & =\frac{\rho g h_{0}}{H_{a}} A_{H}
\end{aligned}
$$

where the stiffness $K_{n}$ of the air spring is found from Eq. (22). With these parameters, we obtain the following equations of motion for the equivalent oscillator:

$$
\left[\begin{array}{cc}
\bar{m}_{f 1} & 0 \\
0 & \bar{m}_{f 2}
\end{array}\right]\left[\begin{array}{l}
\ddot{u}_{1} \\
\ddot{u}_{2}
\end{array}\right]+\left[\begin{array}{cc}
K_{n}+k_{1} & -K_{n} \\
-K_{n} & K_{n}+k_{2}
\end{array}\right]\left[\begin{array}{l}
u_{1} \\
u_{2}
\end{array}\right]=-\left[\begin{array}{ccc}
\kappa_{1} \bar{m}_{f 1} & a_{A 1} \\
\kappa_{2} \bar{m}_{f 2} & a_{A 2}
\end{array}\right],
$$

which, alternatively, can be brought into a form similar to Eq. (18),

$$
\left[\begin{array}{l}
\ddot{u}_{1} \\
\ddot{u}_{2}
\end{array}\right]+\left[\begin{array}{cc}
\frac{g}{L_{01}} & -\frac{g}{L_{\text {effl }}} \frac{h_{0}}{H_{a}} \\
-\frac{g}{L_{\text {eff2 }}} \frac{h_{0}}{H_{a}} & \frac{g}{L_{02}}
\end{array}\right]\left[\begin{array}{l}
u_{1} \\
u_{2}
\end{array}\right]=-\left[\begin{array}{ll}
\kappa_{1} & a_{A 1} \\
\kappa_{2} & a_{A 2}
\end{array}\right],
$$

with

$$
L_{0 k}=\frac{L_{\mathrm{eff} k}}{2 \sin \left(\beta_{k}\right)+h_{0} / H_{a}}, \quad k=1,2 .
$$

\section{TLCGD systems attached to a spatial frame structure}

\subsection{Structural equations}

We consider a frame type building with $N$ horizontal floors under a horizontal ground acceleration $\ddot{\mathbf{x}}_{\mathrm{g}}(t)$ in the $y-z$ plane and a system of $N_{D}$ coupled dampers attached to the $i$-th floor, as shown in Fig. 3. The structure has $3 N$ degrees of freedom $\mathbf{x}=\left[y_{1}, z_{1}, \psi_{1}, y_{2}, z_{2}, \psi_{2}, \ldots, \psi_{N}\right]^{\mathrm{T}}$, where $\psi_{n}$ is the torsional angle of the $n$-th 


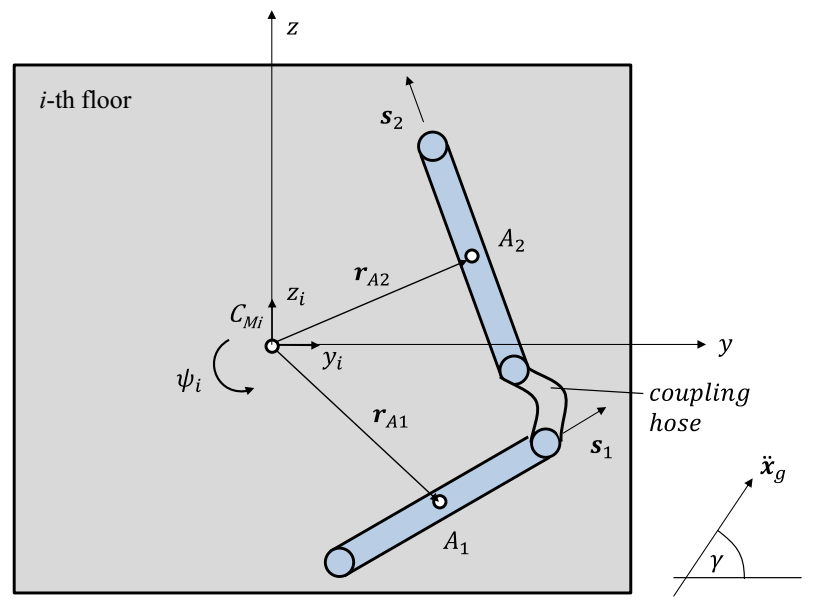

Fig. 3 Two coupled TLCGD attached to the $i$-th floor of a multiple story host structure

floor mass. The origin is chosen to be the center of mass $C_{M i}$ of the host structure. With the corresponding mass, damping and stiffness matrices $\mathbf{M}, \mathbf{C}, \mathbf{K}$, the dynamic equations of the structure read

$$
\mathbf{M} \ddot{\mathbf{x}}+\mathbf{C} \dot{\mathbf{x}}+\mathbf{K} \mathbf{x}=-\mathbf{M} \mathbf{R}_{S} \ddot{\mathbf{x}}_{\mathrm{g}}+\mathbf{F}
$$

where $\ddot{\mathbf{x}}_{\mathrm{g}}=\left[\ddot{y}_{\mathrm{g}}, \ddot{z}_{\mathrm{g}}, 0\right]^{\mathrm{T}}$ is the vector of the ground acceleration, $\mathbf{F}$ denotes the interaction forces between structure and the damping system, and the $(3 N \times 3)$ matrix $\mathbf{R}_{S}$ is given by

$$
\mathbf{R}_{S}=\left[\begin{array}{ccccccc}
1 & 0 & 0 & 1 & 0 & 0 & \ldots \\
0 & 1 & 0 & 0 & 1 & 0 & \ldots \\
0 & 0 & 1 & 0 & 0 & 1 & \ldots
\end{array}\right]^{\mathrm{T}}
$$

The interaction force $\mathbf{F}$ will act on the $i$-th floor, and in case of $N_{D}$ dampers we have

$$
\mathbf{F}=-\mathbf{S}_{i}\left[\mathbf{M}_{f}\left(\ddot{\mathbf{x}}_{\mathrm{g}}+\mathbf{S}_{i}^{\mathrm{T}} \ddot{\mathbf{x}}\right)+\sum_{k=1}^{N_{D}} \bar{\kappa}_{k} m_{f k} \ddot{u}_{k} \mathbf{g}_{k}\right],
$$

with

$$
\begin{aligned}
\mathbf{S}_{i} & =\left[\begin{array}{lllllllllll}
0 & 0 & 0 & \ldots & 1 & 0 & 0 & \ldots & 0 & 0 & 0 \\
0 & 0 & 0 & \ldots & 0 & 1 & 0 & \ldots & 0 & 0 & 0 \\
0 & 0 & 0 & \ldots & 0 & 0 & 1 & \ldots & 0 & 0 & 0
\end{array}\right]^{\mathrm{T}}, \\
\mathbf{M}_{f} & =\sum_{k=1}^{N_{D}} m_{f k}\left[\begin{array}{llll}
1 & 0 & -r_{A k z} \\
0 & 1 & r_{A k y} \\
-r_{A k z} & r_{A k y} & r_{A k}^{2}+i_{k}^{2}
\end{array}\right], \quad i_{k}^{2}=\frac{\Theta_{f k}}{m_{f k}}, \\
\mathbf{g}_{k} & =\left[\begin{array}{c}
s_{k y} \\
s_{k z} \\
\theta_{k}
\end{array}\right] .
\end{aligned}
$$

In Eq. (30.2), $\Theta_{f k}$ is the mass moment of inertia of the fluid with respect to $A_{k}$. The orientation of the $k$-th damper is given by the unit vector $\mathbf{s}_{k}=\left[s_{k y}, s_{k z}\right]^{\mathrm{T}}$, and its center $A_{k}$ is located at $\mathbf{r}_{A k}=\left[r_{A k y}, r_{A k z}\right]^{\mathrm{T}}$, see Fig. 3. With these vectors, we define

$$
\begin{aligned}
r_{A k}^{2} & =\mathbf{r}_{A k} \cdot \mathbf{r}_{A k}, \\
\theta_{k} & =\left(\mathbf{e}_{x} \times \mathbf{r}_{A k}\right) \cdot \mathbf{s}_{k}, \quad k=1, \ldots, N_{D},
\end{aligned}
$$

where $\mathbf{e}_{x}$ is the unit vector in $x$-direction that is normal to the floor plane. 
Eventually, Eq. (27) has to be combined with the dynamic equations of the individual damping devices. For two coupled dampers at the $i$-th floor, we have to use Eq. (24) with the following values of the floor accelerations:

$$
a_{A k}=\mathbf{g}_{k}^{\mathrm{T}}\left(\ddot{\mathbf{x}}_{\mathrm{g}}+\mathbf{S}_{i}^{\mathrm{T}} \ddot{\mathbf{x}}\right), \quad k=1, \ldots, N_{D} .
$$

In both the expressions of $\mathbf{F}$ and $a_{A k}$, we neglect the centripetal components of the acceleration, which due to their dependence on $\dot{\psi}_{i}^{2}$ will be nonlinear. For an oscillatory angle $\psi=A \sin \omega t$, we have a tangential acceleration of the magnitude $\left|a_{t}\right|=|r \ddot{\psi}| \sim\left|r A \omega^{2}\right|$, while the transverse centripetal component reads $\left|a_{n}\right|=\left|(r \dot{\psi})^{2} / r\right| \sim\left|r A^{2} \omega^{2}\right| \sim A\left|a_{t}\right|$. Since the amplitude $A$ of the rotation angle $\psi$ is small, these centripetal terms can safely be ignored.

\subsection{Modal tuning of dampers in spatial structures}

In a first step, we perform a modal decomposition of the host structure, $\mathbf{x}(t)=\sum_{n=1}^{3 N} q_{n}(t) \boldsymbol{\phi}_{n}$. Again, we assume that the structural frequencies are sufficiently well separated. Then, we may restrict the modal host structure to the very two global modes tuned to the damping system. In case of two TLCGD, $N_{D}=2$, we arrive at a four degree of freedom oscillator. If the tuned modes carry the numbers $j 1$ and $j 2$ we may set up the equations for the displacement coordinates $\mathbf{q}=\left[q_{j 1}, q_{j 2}, u_{1}, u_{2}\right]^{\mathrm{T}}$,

$$
\mathbf{M}_{R} \ddot{\mathbf{q}}+\mathbf{C}_{R} \dot{\mathbf{q}}+\mathbf{K}_{R} \mathbf{q}=-\mathbf{E}_{R} \ddot{\mathbf{x}}_{\mathrm{g}}
$$

with the reduced $(4 \times 4)$ matrices

$$
\begin{aligned}
\mathbf{M}_{R}= & {\left[\begin{array}{cccc}
M_{j 1}+\boldsymbol{\phi}_{j 1}^{\mathrm{T}} \mathbf{M}_{f}^{(i)} \boldsymbol{\phi}_{j 1} & \boldsymbol{\phi}_{j 1}^{\mathrm{T}} \mathbf{M}_{f}^{(i)} \boldsymbol{\phi}_{j 2} & \boldsymbol{\phi}_{j 1}^{\mathrm{T}} \mathbf{m}_{f 1}^{(i)} & \boldsymbol{\phi}_{j 1}^{\mathrm{T}} \mathbf{m}_{f 2}^{(i)} \\
\boldsymbol{\phi}_{j 2}^{\mathrm{T}} \mathbf{M}_{f}^{(i)} \boldsymbol{\phi}_{j 1} & M_{j 2}+\boldsymbol{\phi}_{j 2}^{\mathrm{T}} \mathbf{M}_{f}^{(i)} \boldsymbol{\phi}_{j 2} & \boldsymbol{\phi}_{j 2}^{\mathrm{T}} \mathbf{m}_{f 1}^{(i)} & \boldsymbol{\phi}_{j 2}^{\mathrm{T}} \mathbf{m}_{f 2}^{(i)} \\
\mathbf{m}_{f 1}^{(i) \mathrm{T}} \boldsymbol{\phi}_{j 1} & \mathbf{m}_{f 1}^{(i) \mathrm{T}} \boldsymbol{\phi}_{j 2} & \bar{m}_{f 1} & 0 \\
\mathbf{m}_{f 2}^{(i) \mathrm{T}} \boldsymbol{\phi}_{j 1} & \mathbf{m}_{f 2}^{(i) \mathrm{T}} \boldsymbol{\phi}_{j 2} & 0 & \bar{m}_{f 2}
\end{array}\right], } \\
\mathbf{C}_{R}= & \operatorname{diag}\left[\begin{array}{cccc}
2 Z_{j 1} \Omega_{j 1} M_{j 1}, 2 Z_{j 2} \Omega_{j 2} M_{j 2}, 2 \zeta_{1} \omega_{1} \bar{m}_{f 1}, 2 \zeta_{2} \omega_{2} \bar{m}_{f 2}
\end{array}\right], \\
\mathbf{K}_{R}= & {\left[\begin{array}{cccc}
\Omega_{j 1}^{2} M_{j 1} & 0 & 0 & 0 \\
0 & \Omega_{j 2}^{2} M_{j 2} & 0 & 0 \\
0 & 0 & K_{n}+k_{1} & -K_{n} \\
0 & 0 & -K_{n} & K_{n}+k_{2}
\end{array}\right] }
\end{aligned}
$$

where

$$
\begin{aligned}
& \mathbf{M}_{f}^{(i)}=\mathbf{S}_{i} \mathbf{M}_{f} \mathbf{S}_{i}^{\mathrm{T}} . \\
& \mathbf{m}_{f k}^{(i)}=\kappa_{k} \bar{m}_{f k} \mathbf{S}_{i} \mathbf{g}_{k}, \quad k=1,2 .
\end{aligned}
$$

The modal damping coefficients in Eq. (34.2) are usually identified from measured frequency spectra of the structure and the TLCGD system. While $Z_{j 1}, Z_{j 2}$ are predefined values, the coefficients $\zeta_{1}, \zeta_{2}$ of the dampers will be submitted to adaptions for tuning purposes, which we will discuss in Ch. 5. On the right-hand side of Eq. (33), the impact of the ground acceleration on the different degrees of freedom will be governed by the $(4 \times 3)$ matrix

$$
\mathbf{E}_{R}=\left[\begin{array}{c}
\boldsymbol{\phi}_{j 1}^{\mathrm{T}}\left(\mathbf{M} \mathbf{R}_{S}+\mathbf{S}_{i} \mathbf{M}_{f}\right) \\
\boldsymbol{\phi}_{j 2}^{\mathrm{T}}\left(\mathbf{M} \mathbf{R}_{S}+\mathbf{S}_{i} \mathbf{M}_{f}\right) \\
\kappa_{1} \bar{m}_{f 1} \mathbf{g}_{1}^{\mathrm{T}} \\
\kappa_{2} \bar{m}_{f 2} \mathbf{g}_{2}^{\mathrm{T}}
\end{array}\right] .
$$




\section{Optimization and fine-tuning of TLCGD systems in state space}

The Den Hartog criteria of Eq. (17) merely serve as an estimate for single mode tuning and are useful only for well-separated frequencies. This, however, appears to be too restrictive in practical applications. Often, the necessary cross section of the damper tubes turns out to be too large for an individual device. Then, it becomes necessary to split up the device into several small dampers, therefore increasing the number of degrees of freedom. Moreover, a coupled subsystem like Eq. (33) has to include all modes that are likely to interact, which sometimes comprises a significantly large number. Consequently, an optimization procedure has to be applied, which works for an arbitrary number of degrees of freedom. Such a method is usually formulated in state space. With $N_{q}$ structural modes and $N_{D}$ dampers, we have a state vector $\mathbf{z}=[\mathbf{q}, \dot{\mathbf{q}}]^{\mathbf{T}}$, where $\mathbf{q}=\left[q_{j 1}, \ldots, q_{j N_{q}}, u_{1}, \ldots, u_{N_{D}}\right]^{\mathrm{T}}$ contains only the $N_{q} \ll 3 N$ modal coordinates of the reduced-order model along with the damper displacements. The equations of motion in state space read [23]

$$
\dot{\mathbf{z}}=\mathbf{A} \mathbf{z}+\mathbf{B} \ddot{\mathbf{x}}_{\mathrm{g}}
$$

with

$$
\mathbf{A}=\left[\begin{array}{cc}
\mathbf{O} & \mathbf{I} \\
-\mathbf{M}_{R}^{-1} \mathbf{K}_{R} & -\mathbf{M}_{R}^{-1} \mathbf{C}_{R}
\end{array}\right], \quad \mathbf{B}=\left[\begin{array}{c}
\mathbf{0} \\
\mathbf{M}_{R}^{-1} \mathbf{E}_{R}
\end{array}\right] .
$$

Here, the reduced $\left(N_{q D} \times N_{q D}\right)$ matrices $\mathbf{M}_{R}, \mathbf{C}_{R}, \mathbf{K}_{R}$ contain all $N_{q D}=N_{q}+N_{D}$ coupled degrees of freedom, which are relevant for the tuning process.

We assume $\ddot{\mathbf{x}}_{\mathrm{g}}$ to be time harmonic with amplitude $a_{0}$ and forcing frequency $\omega$, directed along the unit vector $\mathbf{e}_{S}=[\cos \gamma, \sin \gamma, 0]^{\mathrm{T}}$, as shown in Fig. 4,

$$
\ddot{\mathbf{x}}_{\mathrm{g}}=a_{0} \mathbf{e}_{S} e^{i \omega t}
$$

Then, the complex steady state solution derived from Eq. (37) reads

$$
\hat{\mathbf{z}}(\omega)=[i \omega \mathbf{I}-\mathbf{A}]^{-1} a_{0} \mathbf{B} \mathbf{e}_{S} .
$$

The optimal tuning parameters result from a minimization of the response over the entire frequency range $\omega$. This is done by minimizing the functional

$$
J=\int_{-\infty}^{\infty} \hat{\mathbf{z}}^{\mathrm{T}}(\omega) \mathbf{S} \hat{\mathbf{z}}(\omega) \mathrm{d} \omega=2 \pi a_{0}^{2} \mathbf{e}_{\mathrm{S}}^{\mathrm{T}} \mathbf{B}^{\mathrm{T}} \mathbf{P} \mathbf{B} \mathbf{e}_{S}
$$

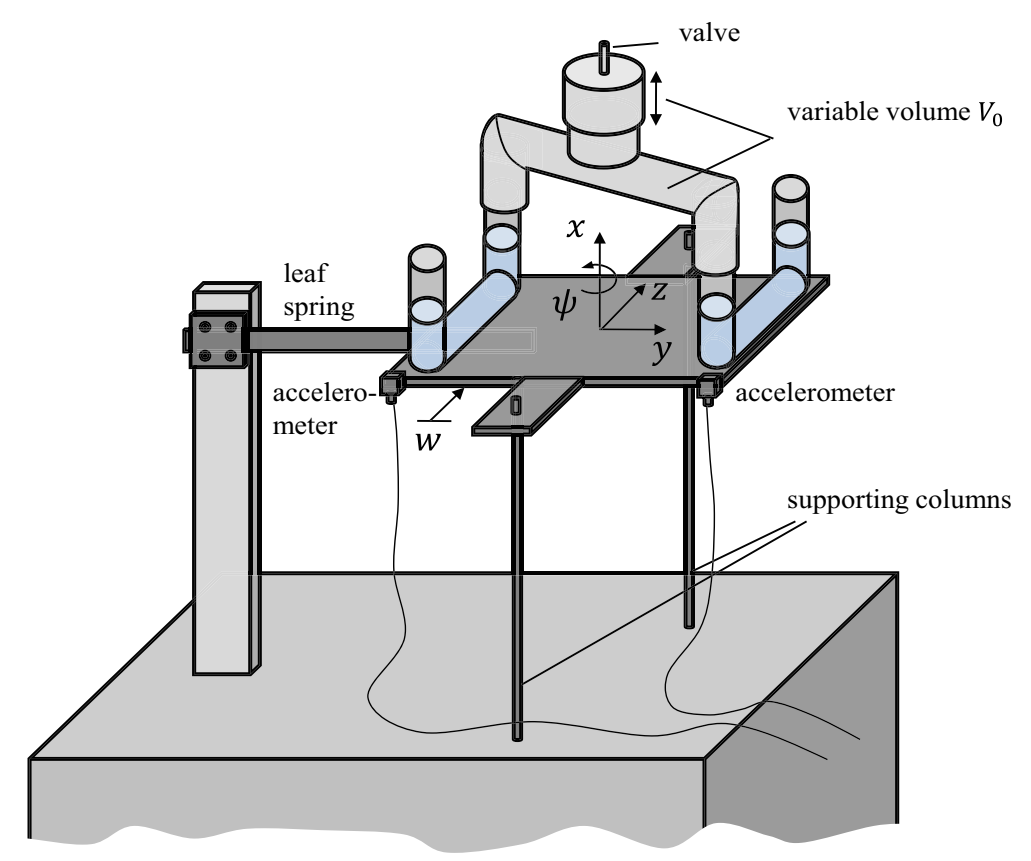

Fig. 4 Two degree of freedom frame, translation $w$ in $z$-direction, rotation $\Psi$ around $x$-axis 
where $\mathbf{S}$ is a $\left(2 N_{q D} \times 2 N_{q D}\right)$ weighting matrix, which accounts for possible differences in the weighting of the states. In the present problem, for example, only the $N_{q}$ modes of the host structure will be regarded in the integral (41), since only their amplitudes are to be reduced. Hence, the weights of the damper amplitudes and velocities in the matrix $\mathbf{S}$ will be zero. The remaining weights are chosen to optimize the structural response with respect to certain system states, e.g., floor displacements. In case of time harmonic excitation, the matrix $\mathbf{P}$ is defined as the solution of the Ljapunov equation, see, e.g., [26],

$$
\mathbf{A}^{\mathrm{T}} \mathbf{P}+\mathbf{P} \mathbf{A}=-\mathbf{S}
$$

The state space response optimization is also important with respect to stochastic vibrations. Then, $\mathbf{P}$ as defined by Eq. (42) is the covariance matrix of the dynamic system, if the excitation is characterized by a stationary random white noise process with zero mean [26]. Since the variance of the system's states is defined by the diagonal elements of $\mathbf{P}$, the covariance matrix can be applied directly for a stochastic optimization by minimizing a properly weighted sum of its diagonal elements.

The formulation of the system's dynamics in state space is very flexible, and it is straightforward to include additional frequency weightings of the cost functional (41), see, e.g., [27]. When filtering the white noise system input before applying it as structural excitation, any colored noise characteristics can be realized by combining the structural system with the filtered input, which increases the system order by the dimension of the corresponding filter. Similarly, it is possible to increase the system dynamics to account for filtered system states. However, if stochastic optimization is performed for optimal absorber tuning, the frequency response function will show minor deviations from a structure optimized according to traditional design guidelines, e.g., Den Hartog tuning. A closer look at the structural resonance characteristics reveals a minor asymmetry compared to well-established design guidelines like Eq. (17), which aim to minimize the response peak only, without taking into account the frequency range outside of resonance. Therefore, it may seem reasonable to minimize the functional with respect to velocities rather than amplitudes, since this puts more weight on higher frequencies. Then, each pair of resonance peaks in the final response spectrum will be leveled more uniformly.

The minimization of the functional $J$, Eq. (41), is performed numerically and delivers the desired primary TLCGD tuning parameters, i.e., the natural frequencies and the damping ratios for a set of independent TLCGD. A proper choice of the initial conditions is obtained by the TMD-TLCGD analogy which renders a preliminary design in accordance with well-established TMD design criteria. If, e.g., an absorber system should be tuned to specific structural modes, the individually selected absorber locations within the host structure must have large displacements of the respective vibration mode for proper performance. For each absorber, a tuning frequency slightly below the structural frequency, and an initial damping ratio dependent on the modal mass ratio $\mu^{*}$, is obtained from the analogy, see Eq. (17). However, in case of coupled TLCGD, the tuning frequencies obtained from the analogy must be in accordance with the natural frequencies of coupled absorber systems defined by Eq. (25), thereby, again, defining basic geometric relations. Nevertheless, in order to find the optimal tuning frequency numerically, the TLCGD geometry must be predetermined to some extent, and commonly the length of the horizontal pipe section $B$, the cross-sectional areas $A_{H}$ and $A_{B}$, as well as the angle $\beta$ are set manually. The coupled TLCGD form a system with several potential design parameters. However, it is beneficial to select those which are easy to adapt in a given setup, e.g., the amount of liquid defined by the liquid column length $H$ or the coupling air spring stiffness $K_{n}$. Since it is not possible to select the modal damping ratios of the coupled TLCGD independently, it is recommended to use the same damping ratio $\zeta$ for each absorber. Adapting $H$ will also ensure that the geometries of both TLCGD remain equal during the optimization process. Depending on the specific problem, though, other combinations of design parameters might be beneficial. It is certainly possible to extend the numerical optimization to include further TLCGD parameters, e.g., the optimal position at a selected floor or the most effective liquid mass distribution when installing several TLCGD. To avoid non-physical solutions, a constrained optimization may be applied, but with the proper initial conditions this will rarely be necessary.

\section{Application to a single-story structure under combined translational and rotational vibrations}

\subsection{Experimental setup}

As an example, we consider a simple spatial frame with two degrees of freedom. The frame may move in a translation along the $z$-axis and rotate around the $x$-axis, while the displacement in the $y$-direction is suppressed by a leaf spring, as shown in Fig. 4. 


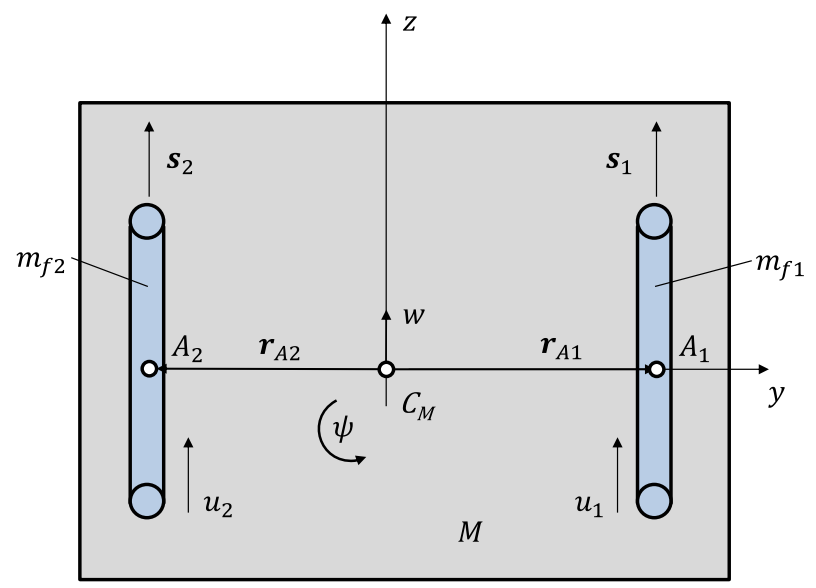

Fig. 5 Two degree of freedom frame, translation $w$ in $z$-direction, rotation $\psi$ around $x$-axis

The two coupled TLCGD are placed collinear to the $z$-axis. The two tubes are diagonally connected by an air chamber with volume $V_{0}$. Hence, a synchronous movement of the liquid might be used to reduce the translational mode, while an out of phase displacement of the fluid level will act on the torsional oscillation. Altogether, this is a four degree of freedom system with $\mathbf{x}=\left[w, \psi, u_{1}, u_{2}\right]^{\mathrm{T}}$. According to the setup shown in Fig. 5, we have

$$
\begin{aligned}
\mathbf{r}_{A 1} & =\left[\begin{array}{c}
r_{A 1} \\
0
\end{array}\right], \mathbf{r}_{A 2}=\left[\begin{array}{c}
-r_{A 2} \\
0
\end{array}\right], \\
\mathbf{g}_{1} & =\left[\begin{array}{c}
0 \\
1 \\
r_{A 1}
\end{array}\right], \mathbf{g}_{2}=\left[\begin{array}{c}
0 \\
1 \\
-r_{A 2}
\end{array}\right], \\
\mathbf{M}_{f} & =\sum_{k=1}^{N_{D}} m_{f k}\left[\begin{array}{cc}
1 & r_{A k y} \\
r_{A k y} & r_{A k}^{2}
\end{array}\right]=\left[\begin{array}{cc}
m_{f 1}+m_{f 2} & r_{A 1} m_{f 1}-r_{A 2} m_{f 2} \\
r_{A 1} m_{f 1}-r_{A 2} m_{f 2} & r_{A 1}^{2} m_{f 1}+r_{A 2}^{2} m_{f 2}
\end{array}\right] .
\end{aligned}
$$

With these parameters, we obtain for the mass matrix $\mathbf{M}_{R}$

$$
\mathbf{M}_{R}=\left[\begin{array}{cccc}
M+m_{f 1}++m_{f 2} & r_{A 1} m_{f 1}-r_{A 2} m_{f 2} & \kappa_{1} \bar{m}_{f 1} & \kappa_{2} \bar{m}_{f 2} \\
r_{A 1} m_{f 1}-r_{A 2} m_{f 2} & \Theta+r_{A 1}^{2} m_{f 1}+r_{A 2}^{2} m_{f 2} & \bar{\kappa}_{1} m_{f 1} r_{A 1} & -\kappa_{2} \bar{m}_{f 2} r_{A 2} \\
\kappa_{1} \bar{m}_{f 1} & \kappa_{1} m_{f 1} r_{A 1} & \kappa_{1} \bar{m}_{f 1} & 0 \\
\kappa_{2} \bar{m}_{f 2} & -\kappa_{2} \bar{m}_{f 2} r_{A 2} & 0 & \kappa_{2} \bar{m}_{f 2}
\end{array}\right]
$$

where $M_{1}=M$ and $M_{2}=\Theta$ are the story's mass and mass momentum of inertia, respectively. In Eq. (43.3), we neglected $i_{k}^{2}$ compared to $r_{A k}^{2}$, cf. Eq. (30.2).

We assume both TLCGD to be equal in size and fluid mass and their location to be symmetric with respect to the mass center $C_{M}$, that is, $m_{f 1}=m_{f 2}, \mathbf{s}_{1}=\mathbf{s}_{2}=[0,1]^{\mathrm{T}}, r_{A 1}=r_{A 2}$. Consequently, the first damper mode will be tuned to the translational mode of the structure, while the second damper mode is tuned to the torsional mode. Only the second mode activates the gas spring between the tubes, and therefore, the volume $V_{0}$ can be used only for the damping of the rotational oscillations.

\subsection{Structural parameters}

The relevant parameters of the host structure are given in Table 1. The natural frequencies $\Omega_{1}$ and $\Omega_{2}$ were identified from Fourier spectra of the free vibration, and the corresponding translational and rotational mode shapes are fully decoupled and shown in Fig. 6.

The geometrical properties of the damping system are given by 
Table 1 Parameters of the host structure

\begin{tabular}{lll}
\hline & $j=1$ & $j=2$ \\
\hline$M_{j}$ & $4.62 \mathrm{~kg}$ & $8.12 \times 10^{-2} \mathrm{~kg} \mathrm{~m}{ }^{2}$ \\
$\Omega_{j}$ & $7.38 \mathrm{rad} / \mathrm{s}$ & $14.14 \mathrm{rad} / \mathrm{s}$ \\
$Z_{j}$ & 0.0072 & 0.0113 \\
\hline
\end{tabular}

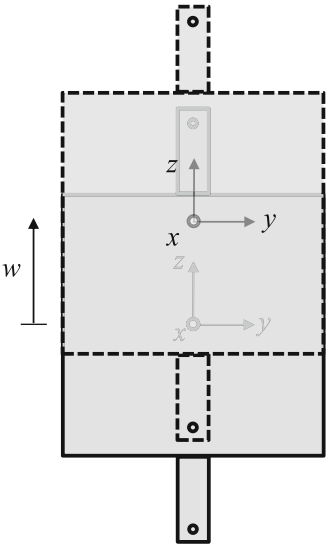

(a)

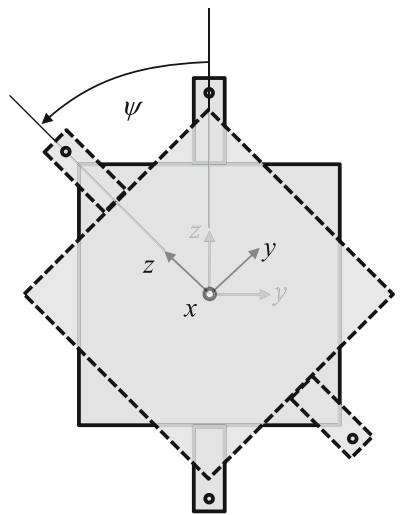

(b)

Fig. 6 Fully decoupled mode shapes of host structure a bending mode $\Omega_{1}$, b torsional mode $\Omega_{2}$

$$
r_{A 1}=r_{A 2}=0.125 \mathrm{~m}, \quad \beta_{1}=\beta_{2}=\pi / 2
$$

The frequency and the damping coefficient of the absorber are to be found from an optimization procedure according to $\mathrm{Ch}$. 5 with the diagonal weighting matrix $\mathbf{S}=\operatorname{diag}(0,0,0,0,1,0.01,0,0)$ selected to minimize $\dot{w}$ and $\dot{\psi}$. During the optimization of the amount of liquid, its damping as well as the air spring volume $V_{0}$ are optimized, still assuming identical parameters for both TLCGD. For the present host structure with identical dampers, we obtain the individual values of frequency and damping ratio as

$$
\begin{aligned}
\omega_{01} & =\omega_{02}=7.15 \mathrm{rad} / \mathrm{s}, \\
\zeta_{01} & =\zeta_{02}=13.90 \times 10^{-2} .
\end{aligned}
$$

Now it will be important to achieve desired values of $\omega$ and $\zeta$ for the coupled TLCGD system, since they have to match the properties of the relevant structural modes. The corresponding results are

$$
\begin{aligned}
\omega_{1} & =7.15 \mathrm{rad} / \mathrm{s}, \quad \omega_{2}=14.04 \mathrm{rad} / \mathrm{s}, \\
\zeta_{1} & =13.90 \times 10^{-2}, \quad \zeta_{2}=7.07 \times 10^{-2} .
\end{aligned}
$$

Since both dampers are identical and collinear, they move synchronously in the first mode where the air spring will not be activated. Hence, $\omega_{1}$ and $\zeta_{1}$ of the coupled system are equal to the corresponding values of the individual damper in Eq. (46).

From the optimization, one obtains a liquid mass of $m_{\mathrm{f}}=0.270 \mathrm{~kg}$ for each tube. The corresponding parameters are summarized in Table 2, which holds for both dampers.

Table 2 Parameters of the dual TLCGD system

\begin{tabular}{llllll}
\hline$m_{\mathrm{f}}(\mathrm{kg})$ & $L_{\mathrm{eff}}(\mathrm{m})$ & $\kappa$ & $\bar{\kappa}$ & $h_{0} / H_{a}$ & $L_{0}(\mathrm{~m})$ \\
\hline 0.270 & 0.40 & 0.85 & 0.68 & 2.19 & 0.20 \\
\hline
\end{tabular}




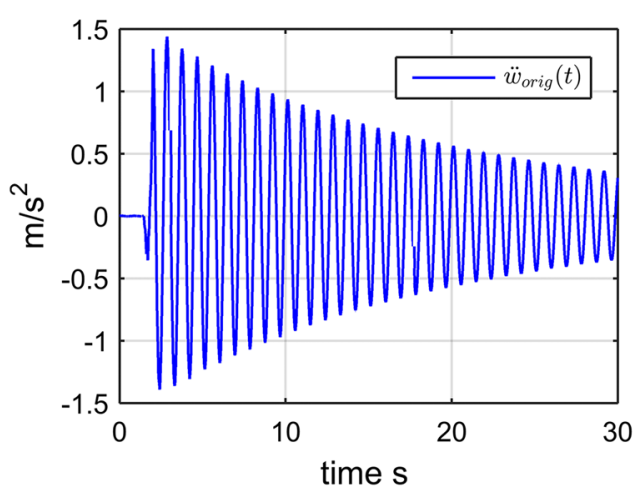

(a)

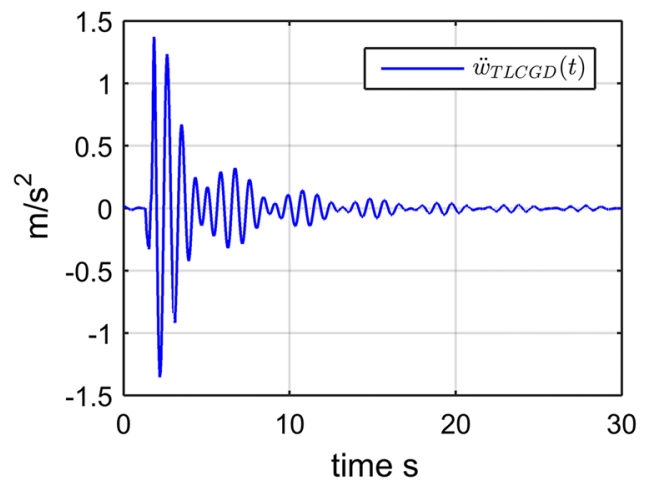

(c)

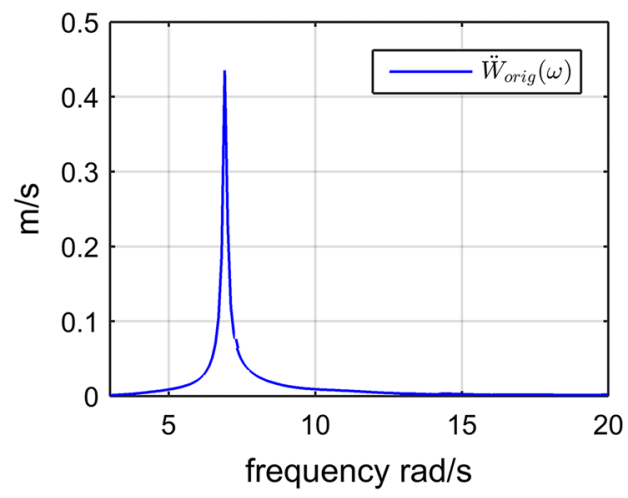

(b)

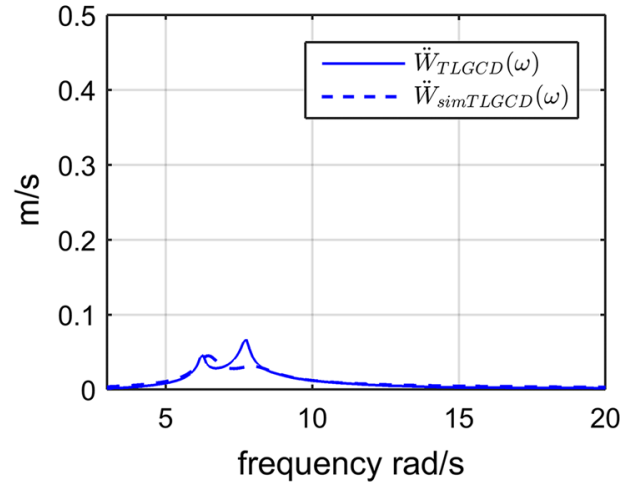

(d)

Fig. 7 Acceleration measurement (mode 1) of free decay $\ddot{w}(t)$ of the two degree of freedom host structure. a, b Time response $\ddot{w}(t)$ and spectrum $\ddot{W}(\omega)$ of the original system. c, d Time and spectral response of the system with coupled TLCGD attached. The dashed line in $\mathbf{7} \mathbf{d}$ shows the simulated results for comparison

\subsection{Results}

The performance of the damping system is investigated numerically and compared to experimental results carried out in [28]. The latter are recorded from measurements by accelerometers applied at the platform of the structure, as shown in Fig. 5. Starting from the design criteria defined in Ch. 6.2, the experimental fine-tuning yields a final liquid mass of $m_{\mathrm{f}}=0.274 \mathrm{~kg}$.

\subsubsection{Experimental results}

The excellent performance of the final configuration is demonstrated in a free decay experiment, where the initial structural displacements, $w(0)=0.03 \mathrm{~m}$ and $\psi(0)=0 \mathrm{rad}$, correspond to the first mode shape and excite the translational mode only. For the liquid columns initially in equilibrium $u_{1}(0)=u_{2}(0)=0 \mathrm{~m}$, the time response and the spectrum of the acceleration $\ddot{w}(t)$ of the original system with the water mass added are given in Fig. 7a, b. The measurement is repeated with the optimally coupled TLCGD installed, as shown in Fig. 7c, d. When compared to the original system, the increase in damping and the improvement in the dynamic behavior is apparent in both the time response $\ddot{w}(t)$ and its spectrum $\ddot{W}(\omega)$. In Fig. 7d, also the simulated result of the spectral response is shown for comparison. A satisfactory coincidence can be observed. Numerical results in the time domain are given in Ch. 6.3.2.

The free decay test is also performed with initial conditions exciting the torsional mode of vibration only, see Fig. 8a, b. To obtain this second mode excitation, the initial structural displacements are chosen affine to the second mode shape, $w(0)=0 \mathrm{~m}$ and $\psi(0)=0.12 \mathrm{rad}$, with the liquid columns in equilibrium condition, $u_{1}(0)=u_{2}(0)=0 \mathrm{~m}$. Again, the response characteristics of a lightly damped resonance can be identified. Repeating the experiment with the optimally tuned coupled TLCGD attached results in the expected free decay response of a dynamic system with an absorber tuned to a single mode, as shown in Fig. 8c, d. Again 


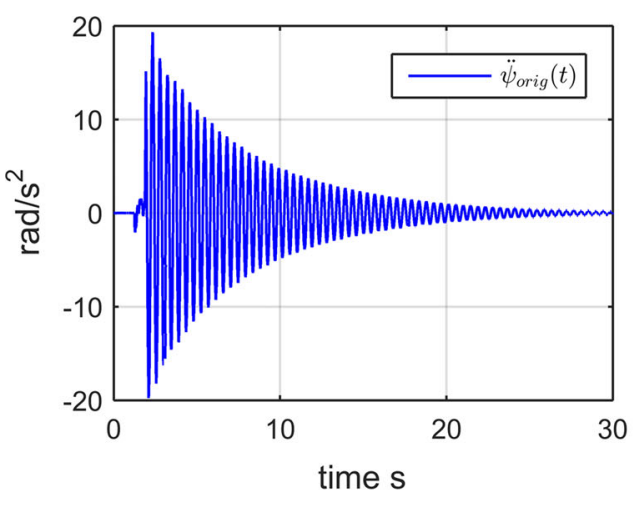

(a)

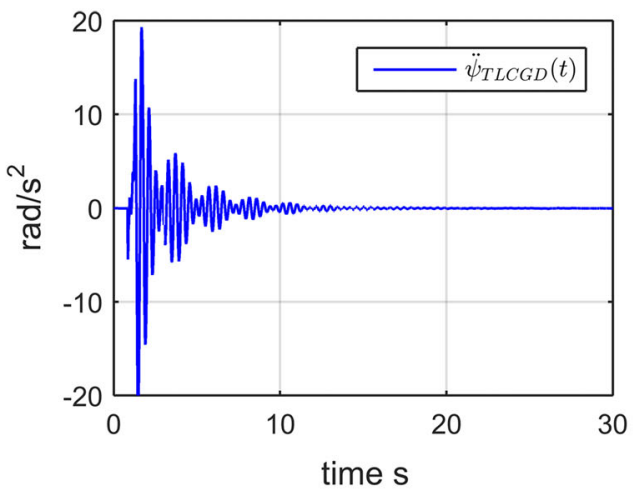

(c)

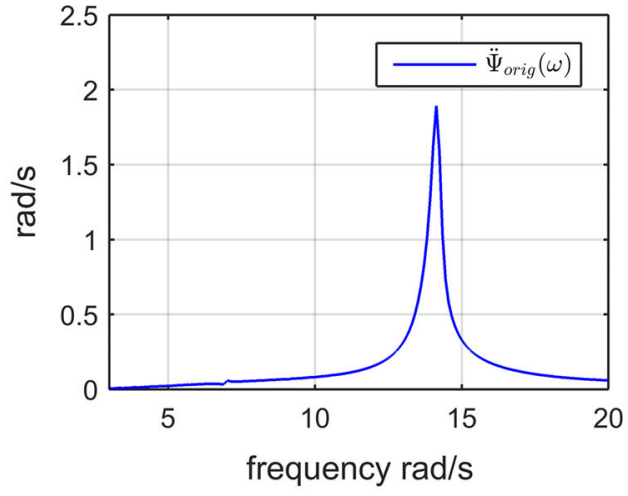

(b)

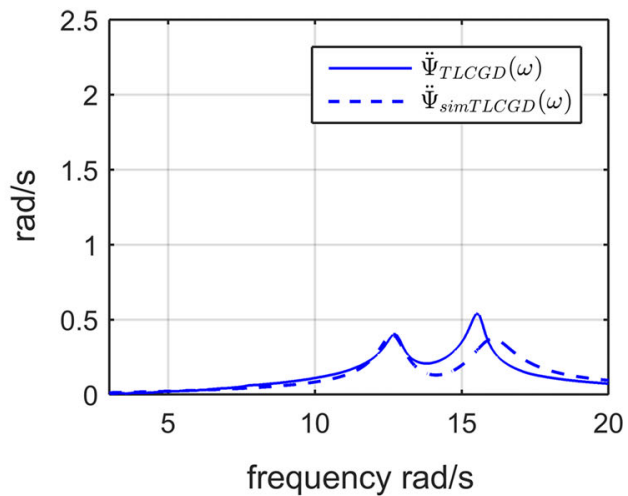

(d)

Fig. 8 Angular acceleration measurement (mode 2) of free decay $\psi(t)$ of two degree of freedom host structure. a, b Time response $\ddot{\psi}(t)$ and spectrum $\ddot{\Psi}(\omega)$ of original system. $\mathbf{c}, \mathbf{d}$ Time and spectral response of system with coupled TLCGD attached. The dashed line in Fig. 8d shows the simulated results for comparison

an acceptable coincidence between experimental and calculated results is found for the angular acceleration spectrum, as shown in Fig. 8d.

Since both individual modes show the desired damping behavior a final measurement is performed to demonstrate the simultaneous suppression of multi-mode excitation. This time the initial displacement is given by an initial translation with a superimposed rotation. The free decay spectrum, as shown in Fig. 9, proves clearly that the proposed damping device is able to reduce both modes of vibration at the same time.

The experiments also demonstrate that the concept of the passive air spring is well suited for tuning the TLCGD to frequencies hard to implement with traditional design. Depending on the geometry of the coupling volume, it is possible to separate $V_{0}$ into sections connected by elements with adjustable pneumatic resistances, e.g., the vertical cylindrical reservoir in Fig. 4. In this case, the choice of a suitable geometry allows modifications in the damping of the higher mode independent from the selected fluid damping for optimal suppression of the fundamental resonance. However, this type of additional damping is not applied in the current experiment, and therefore, the damping coefficient $\zeta_{2}$ is significantly lower than $\zeta_{1}$, see Eq. (47).

\subsubsection{Numerical results}

For comparison, the free decay response of mode 1 is also analyzed numerically. Only graphs in the time domain are presented. Confrontations of the spectral components were already shown in Figs. 7d and 8d. As expected, the simulation of the structural response shows excellent agreement with the experiment for both the original system and the structure with the TLCGD installed, see Fig. 10a-d. However, a numerical simulation also allows the estimate of quantities that are almost impossible to measure directly, e.g., the TLCGD liquid surface accelerations. The results reveal that the liquid columns are moving in phase at the low frequency translational mode, whereas they oscillate in anti-phase when counteracting the torsional mode, see Fig. 10e-f. 


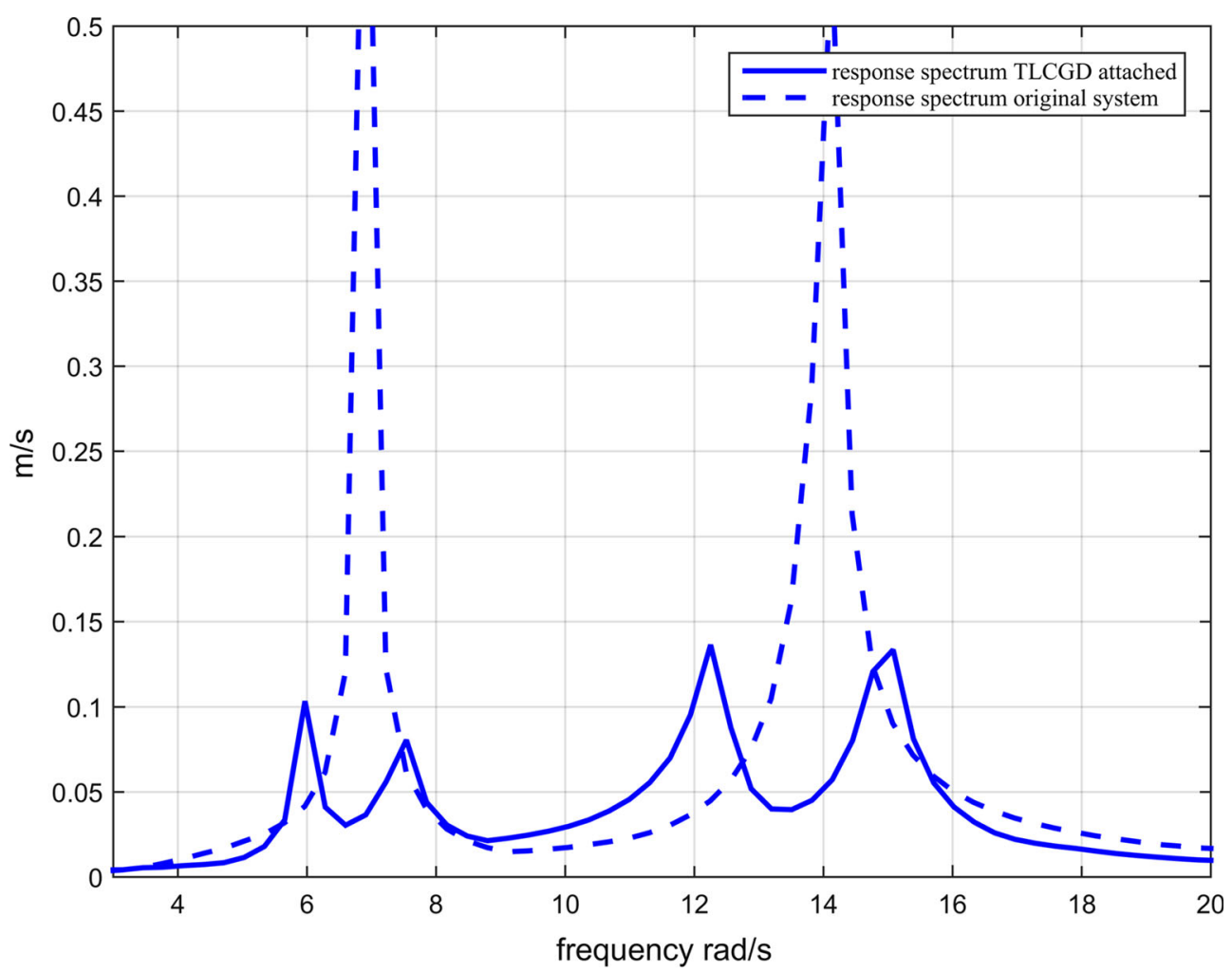

Fig. 9 Experimental measurements of the response spectrum of combined excitation for the two degree of freedom frame. Dashed line: original system without TLCGD. Solid line: host structure response with attached TLCGD

\section{Conclusions}

Tuned liquid column gas dampers (TLCGD) show excellent vibration and energy absorbing capabilities and turn out to be appropriate for various applications in earthquake engineering. In many of the cited papers, it has been shown by Prof. Ziegler and his co-workers that the vibration reduction is competitive to TMD-type absorbers. Contrary to the latter, they do not rely on movable and flexible parts, and their design allows a virtually unlimited number of possible configurations. Since the moving mass is a liquid, the device itself consists of a fixed container only and is therefore easy to build and cost-effective. For practical purposes, the liquid column length has to be in the range of several meters up to thirty meters and more for large structures, causing the classical TLCD's natural frequency to be far below $1 \mathrm{~Hz}$. Due to the incorporation of the gas springs by sealed and pressurized gas volumes, the practical frequency range can be increased to several $\mathrm{Hz}$. The application field of TLCGD can be further enlarged by coupling several devices into a spatial chain of multiple absorbers, representing a secondary multi-degree of freedom system that can be tuned to a selected number of structural modes. Typically, a conventional TLCGD is designed to counteract a single mode only, where a good estimate of the damper parameters is given by Den Hartog's formulas. By installing coupled TLCGD, it becomes possible to reduce several modes simultaneously, even for different types of motions, such as translation and torsion.

Frequently, it is sufficient to consider only a coupling between the absorbers and the structural modes to be reduced. This leads to a considerable reduction in the dimension of the remaining dynamic system. Then, a numerical tuning process yields an optimal set of parameters for the damping device. Such an optimization can be carried out for steady state vibrations as well as for stochastic inputs.

The innovation of the proposed design lies in the substantial increase in active liquid mass, because all absorbers vibrate at different natural frequencies at the same time. This extends the limits of conventional TLCGD which are only able to counteract a single mode of vibration. A simple example as discussed in Ch. 6 is sufficient to demonstrate the capabilities of the proposed damping device in practical applications. Furthermore, it illustrates that the concept of coupled TLGCD is adequate for base-isolated structures if the dampers are tuned to the corresponding fundamental modes. The application of TLCGD is by no means 


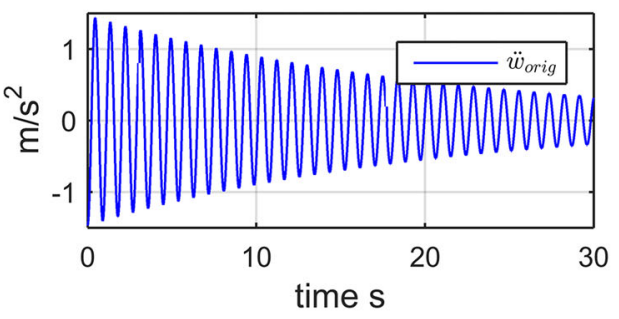

(a)

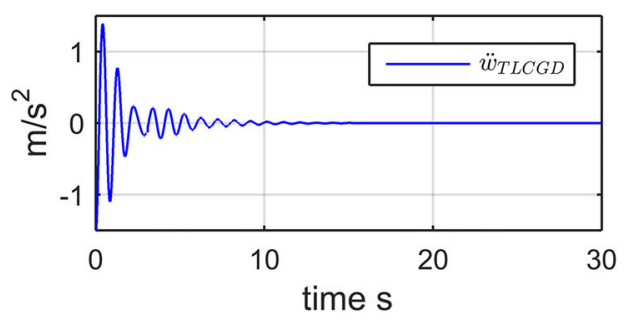

(c)

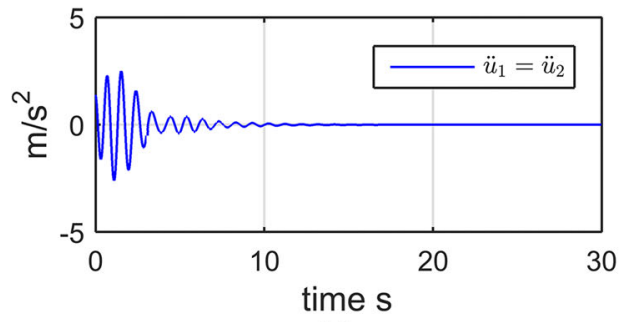

(e)

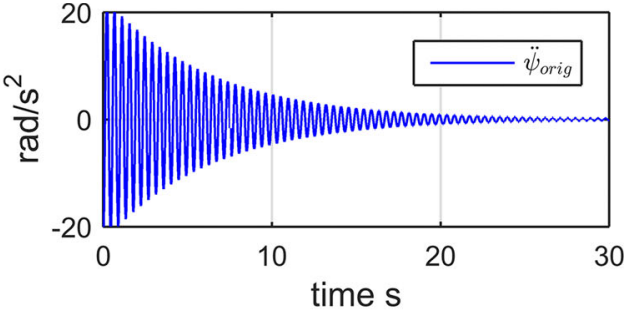

(b)

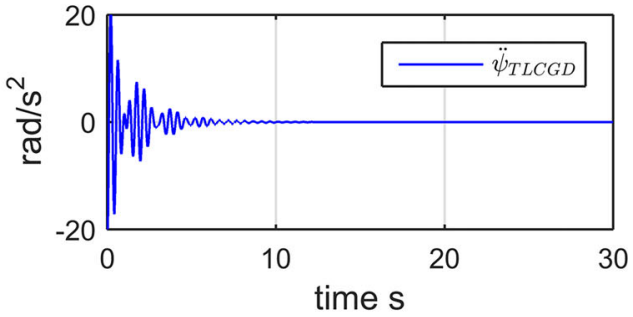

(d)

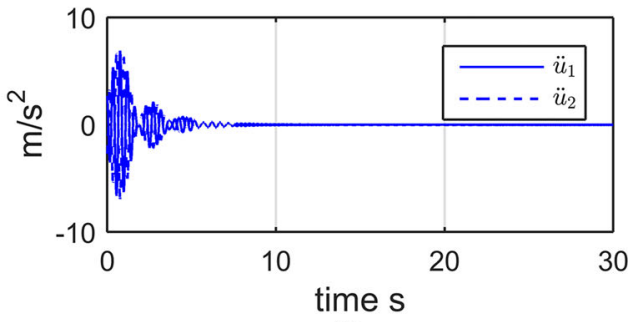

(f)

Fig. 10 Two degree of freedom frame, simulated free decay for translational and rotational modal response. a, b Original structure without coupled TLCGD, $\mathbf{c}, \mathbf{d}$ structure with TLCGD attached, e, $\mathbf{f}$ absorber response. Left: translational mode, right: rotational mode

limited to frame type buildings but may be applied to all structures with dominant natural frequencies in the range of $0-5 \mathrm{~Hz}$. For a study of different applications of such devices in civil engineering, see especially Ref. [9].

Acknowledgements Open access funding provided by University of Applied Sciences Wiener Neustadt for Business and Engineering.

Open Access This article is distributed under the terms of the Creative Commons Attribution 4.0 International License (http:// creativecommons.org/licenses/by/4.0/), which permits unrestricted use, distribution, and reproduction in any medium, provided you give appropriate credit to the original author(s) and the source, provide a link to the Creative Commons license, and indicate if changes were made.

\section{References}

1. Di Matteo, A., Lo Iacono, F., Navarra, G., Pirrotta, A.: Innovative modeling of tuned liquid column damper motion. Commun. Nonlinear Sci. Numer. Simul. 23, 229-244 (2015)

2. Di Matteo, A., Lo Iacono, F., Navarra, G., Pirrotta, A.: Optimal tuning of tuned liquid column damper systems in random vibration by means of an approximate formulation. Meccanica 50, 795-808 (2015)

3. La Duc, V., Adam, C.: General on-off damping controller for semi-active tuned liquid column damper. J. Vib. Control (2016). https://doi.org/10.1177/1077546316648080

4. Hochrainer, M.J.: Control of vibrations of civil engineering structures with special emphasis on tall buildings. Doctoral dissertation, Vienna University of Technology, Austria (2001). http://www.ub.tuwien.ac.at/diss/AC03322409.pdf

5. Fu, C., Ziegler, F.: Vibration prone multi-purpose buildings and towers effectively damped by tuned liquid column-gas dampers. Asian J. Civ. Eng. 10(1), 21-56 (2010)

6. Reiterer, M.: Damping of vibrational civil engineering structures with emphasis on bridges. Doctoral dissertation, Vienna University of Technology, Austria (2004). http://www.ub.tuwien.ac.at/diss/AC04250350.pdf(in German)

7. Reiterer, M., Ziegler, F.: Bi-axial seismic activation of civil engineering structures equipped with tuned liquid column dampers. J. Seismol. Earthq. Eng. 6(3), 45-60 (2004) 
8. Ziegler, F.: Special design of tuned liquid column-gas dampers for the control of spatial structural vibrations. Acta Mech. 201(1), 249-267 (2008)

9. Hochrainer, M.J., Ziegler, F.: Tuned liquid column gas damper in structural control: the salient features of a general purpose damping device and its application in buildings, bridges and dams. In: Lagaros, N.D., et al. (eds.) Design Optimization of Active and Passive Structural Control Systems, ch. 7, pp. 150-179. IGI Global, Hershey (2013)

10. Ziegler, F., Khalid, B.: A novel base isolation system for asymmetric buildings in seismic active zones: damping supplied by tuned liquid column-gas dampers. In: Belyaev, A.K., Irschik, H., Krommer, M. (eds.) Advanced Dynamics and Model Based Control of Structures and Machines, pp. 225-234. Springer, Wien (2011)

11. Khalid, B., Ziegler, F.: Experimental verification of control of asymmetric building vibrations by tuned liquid column gas dampers. In: Proceedings of the 3rd International Conference on Experiments/Process/System Modeling/Simulation and Optimisation. Athens, 8-11 July, 2009. http://epsmso.gr/2009/default.htm, CD-ROM paper: epsmso09_142.pdf (2009)

12. Khalid, B.: Control of seismically forced vibrations of asymmetric buildings by means of a novel base isolation system. Doctoral dissertation, Vienna University of Technology, Austria (2010). http://www.ub.tuwien.ac.at/diss/AC07809200.pdf

13. Khalid, B., Ziegler, F.: A novel aseismic foundation system for multipurpose asymmetric buildings. Arch. Appl. Mech. 82(10), 1423-1437 (2012)

14. Fu, C.: Effective damping of vibrations of plan-asymmetric buildings. Doctoral dissertation, Vienna University of Technology, Austria (2008). http://www.ub.tuwien.ac.at/diss/AC05037516.pdf

15. Hochrainer, M.J.: Experimental investigations of active tuned liquid column gas damper. In: Vienna Congress on Recent Advances in Earthquake Engineering and Structural Dynamics 2013 (VEESD 2013), 28-30 August 2013, Vienna, Austria, Paper No. 142 (2013)

16. Hochrainer, M.J.: Active tuned liquid column gas damper in structural control. In: Dynamics of Civil Structures, vol. 2, pp. 467-473. Proceedings of the 33rd IMAC, 978-3-319-15248-6 (2015)

17. Reiterer, M., Ziegler, F.: Control of pedestrian-induced vibrations of long-span bridges. Struct. Control Health Monit. 13, 1003-1027 (2006)

18. Teramura, A., Yoshida, O.: Development of vibration control system using U-shaped water tank. In: Proceedings of 11th World Conference on Earthquake Engineering (Sociedad Mexicana de Ingenieria Sismica, A.C.), paper no. 1343. Elsevier, Amsterdam (1996)

19. RWDI Consultant Engineers: Projects from RWDI. Retrieved March 28, 2011 from http://www.rwdi.com/project/one_wall_ centre (2011)

20. Tributsch, A., Adam, C.: Evaluation and analytical approximation of tuned mass damper performance in an earthquake environment. Smart Struct. Syst. 10, 155-179 (2012)

21. Ziegler, F.: Mechanics of Solids and Fluids, 2nd edn. Springer, New York (1998)

22. Hochrainer, M.J.: Tuned liquid column damper for structural control. Acta Mech. 175(1-4), 57-76 (2005)

23. Hochrainer, M.J., Ziegler, F.: Control of tall building vibrations by sealed tuned liquid column dampers. Struct. Control Health Monit. 13(6), 980-1002 (2006)

24. Den Hartog, J.P.: Mechanical Vibrations, reprint of 4th edn. McGraw-Hill, New York (1956)

25. Warburton, G.B.: Optimum absorber parameters for various combinations of response and excitation parameters. Earthq. Eng. Struct. Dyn. 10, 381-401 (1982)

26. Müller, P.C., Schiehlen, W.O.: Lineare Schwingungen. Akademische Verlagsgesellschaft, Wiesbaden (1976)

27. Levine, W.S. (ed.): The Control Handbook, 2nd edn. CRC Press, Tayor and Francis Group, Boca Raton (2011)

28. Ringhofer, B.: Experimental and analytical investigations of passive liquid column dampers in vibration isolated structures. Master thesis, University of Applied Sciences, Wiener Neustadt (2011) (in German)

29. Di Matteo, A., Furtmüller, T., Adam, C., Pirrotta, A.: Optimal design of tuned liquid column dampers for seismic response control of base-isolated structures. Acta Mech (2017). https://doi.org/10.1007/s00707-017-1980-7 